\title{
Assessment of Apple (Malus pumila Mill.) germplasm in Kashmir III. Cytology of Lal-farashi, Double-Kaseri, Hindwand- rakam, Kichhama-trail, Sabe-alif and Tursh-nawabi
}

\author{
Ranbir Singh, B. A. Wafai and A. K. Koul \\ Department of Biosciences, University of Jammu, \\ Jammu-180001, India
}

Accepted June 12, 1984

Sixty five triploid varieties have so far been reported in Malus pumila (Roscoe 1934, Einset and Imhofe 1947, 1949, Einset and Lamb 1951, Hunter and Walker 1955, Shanti et al. 1963, Hathwala 1966, Pratt et al. 1978). Recently, Koul et al. (in press) and Singh and Wafai (in press) have listed four triploids from Kashmir Valley. Further exploration has brought to light six more. The pressent communication describes the morphology and pollen mother cell meiosis of these triploids with a view to assess the impact of triploidy on their floral and fruit characteristics and discuss the nature of triploidy.

\section{Material and methods}

Six varieties, namely Lal-farashi, Double-kaseri, Hindwandrakam, Kichhamatrail, Sabe-alif and Tursh-nawabi constitute the material for investigation. The observations are based on trees growing in different orchards listed in Table on next. page. cedure.

Meiotic studies have been undertaken using Lespinasse et al.'s (1976) pro-

\section{Observations}

\section{Morphological descriptions}

1.1 Lal-farashi: Tree medium to large sized with widespreading open head; branches drooping; flowers pinkish-white, $4.4-6.0 \mathrm{~cm}$ across, in clusters of 3-7; fruit dull yellow, striped in saffron, spherical to oblate, symmetric, sometimes angular, not very uniform, 4.5-6.5 $\times 5.9-7.2 \mathrm{~cm}$; skin thin, rough; lenticels large, brown, raised, conspicuous, concentrated around the apex of fruit; fruit pedicel greenish-brown, pubescent, $2.4-2.9 \mathrm{~cm}$ long, $0.2 \mathrm{~cm}$ thick; stem cavity acute or nearly acuminate, occasionally lipped, heavily lined with golden yellow russet, $2.5-3.0 \mathrm{~cm}$ wide, 1.4 $1.8 \mathrm{~cm}$ deep; basin narrow, gradual, regular, symmetric, russeted in brown, 2.4 $2.7 \mathrm{~cm}$ wide, $0.2-0.3 \mathrm{~cm}$ deep; calyx reflexed, flattened, lobes pubescent; eye mostly closed, occasionally open; calyx tube cone shaped, $1.0-1.2 \mathrm{~cm}$ deep, $0.6-0.8 \mathrm{~cm}$ wide; stamens persistent, placed at the base of calyx tube; pistil point absent; core median or slightly distant, closed, $3.5-4.0 \mathrm{~cm}$ wide; core lines clasping; fruit flesh greenish 
yellow, hard, juicy, subacidic; seed cavities 5 , axile, round or cordate; seeds 1-3 per cavity, $7-10$ per fruit, obtuse or nearly acute, $0.8-1.0 \times 0.5-0.6 \mathrm{~cm}$; fruit yield low to medium; trees neither strict annuals nor strict biennials; fruit production is good in alternate years; blooming period mid April, duration of bloom 6-8 days; fruit ripens within 160-170 days and is harvested in early October.

1.2. Double-kaseri: Trees small, semi erect with open head; flowers in clusters of 4-6, wihte with pink splashes, petals leathery; flower spread $4.7-5.0 \mathrm{~cm}$; fruit uniformly deep red, oblate, generally lopsided, angular, occasionally symmetric, 4.8-6.2 $\times 6.0-7.4 \mathrm{~cm}$; skin thin, tough, smooth, oily; lenticels small, white with a red ring around, depressed or submerged, concentrated towards fruit apex; fruit pedicel green, pubescent, $0.8-1.0 \mathrm{~cm}$ long, $0.2-0.3 \mathrm{~cm}$ thick; stem cavity acute to acuminate, heavily russeted, $2.5-3.0 \mathrm{~cm}$ wide, $1.3-1.5 \mathrm{~cm}$ deep; basin gradual, regular, symmetric, $2.3-2.7 \mathrm{~cm}$ wide, $0.4-0.6 \mathrm{~cm}$ deep; eye open; calyx tube cone or

The list of trees observed

\begin{tabular}{|c|c|c|}
\hline $\begin{array}{l}\text { Name of the } \\
\text { variety }\end{array}$ & $\begin{array}{c}\text { Tree } \\
\text { number }\end{array}$ & Orchard \\
\hline Lal-farashi & $\begin{array}{l}\mathrm{LF}_{1}, \mathrm{LF}_{2} \\
18^{*}\end{array}$ & $\begin{array}{l}\text { 1. Malik Orchards, Khounmoh, Kashmir } \\
\text { 2. Nehru Memorial Botanical Gardens, Chasma } \\
\text { Shahi, Srinagar, Kashmir }\end{array}$ \\
\hline Double-kaseri & $\mathrm{DK}_{1}$ & Malik Orchards, Khounmoh, Kashmir \\
\hline Hindwand-rakam & $2545^{*}$ & $\begin{array}{l}\text { Jyoti Pvt. Ltd; Orchards, Boulevard, Srinagar, } \\
\text { Kashmir }\end{array}$ \\
\hline \multirow[t]{2}{*}{ Kichhama-trail } & $27^{*}$ & $\begin{array}{l}\text { 1. Nehru Memorial Botanical Gardens, Chasma } \\
\text { Shahi, Srinagar, Kashmir }\end{array}$ \\
\hline & $1525^{*}$ & $\begin{array}{l}\text { 2. Jyoti Pvi. Ltd; Orchards, Boulevard, Srinagar, } \\
\text { Kashmir }\end{array}$ \\
\hline Sabe-alif & $34 *$ & $\begin{array}{l}\text { Nehru Memorial Botanical Gardens, Chasma } \\
\text { Shahi, Srinagar, Kashmir }\end{array}$ \\
\hline \multirow[t]{3}{*}{ Tursh-nawabi } & $20^{*}$ & $\begin{array}{l}\text { 1. Nehru Memorial Botanical Gardens, Chasma } \\
\text { Shahi, Srinagar, Kashmir }\end{array}$ \\
\hline & $3884^{*}$ & $\begin{array}{l}\text { 2. Jyoti Pvt. Ltd; Orchards, Boulevard, Srinagar, } \\
\text { Kashmir }\end{array}$ \\
\hline & $\mathrm{TN}_{1}$ & 3. Malik Orchards, Khounmoh, Kashmir \\
\hline
\end{tabular}

* The numbers have been given to individual trees by the orchardists.

almost funnel shaped, $0.5-0.7 \mathrm{~cm}$ wide, $0.5-0.6 \mathrm{~cm}$ deep; stamens median in the calyx tube; pistil-point absent; core median, closed, $3.6-4.0 \mathrm{~cm}$ wide; corelines clasping or nearly meeting; fruit flesh quite firm, hard, light green, quite juicy, crisp, sweet; seed cavities 5, obcordate, axile; seeds none to one per cell, 3-5 per fruit, mostly aborted, acute, $0.8-0.9 \times 0.5 \mathrm{~cm}$ in size; trees biennial; fruit yield very low; blooming period late April; duration of flowering 10-12 days; fruit ripens within 150-160 days; harvesting period late September or early October.

1.3. Hindwand-rakam: Trees small, semierect with small open head; flowers pinkish white, in clusters of 3-7; flowers $3.2-4.2 \mathrm{~cm}$ across; fruit yellow, oblate, compressed, ribbed, oblique, irreguar, angular, not uniform, $6.1-6.5 \times 7.2-8.4 \mathrm{~cm}$; skin thick, smooth, waxy; skin lenticels large, areolar, initially submerged or depressed but brown, raised, open, rough at maturity, concentrated towards the apex 
of fruit; fruit pedicel thick, fleshy, dull green, pubescent, clubbed in the cavity; stem cavity obtuse or nearly acute, occasionally lipped, $2.8-3.5 \mathrm{~cm}$ wide, $0.8-1.2 \mathrm{~cm}$ deep; basin abrupt, obtuse, symmetric, $2.0-2.5 \mathrm{~cm}$ wide, $0.4-0.5 \mathrm{~cm}$ deep; calyx persistent, connivent, pubescent; eye closed or partly open; calyx tube funnel shaped, $0.5-0.6 \mathrm{~cm}$ broad, $1.2-1.4 \mathrm{~cm}$ deep; persistent stamens basal; pistil point absent; core median, open, $4.2-4.5 \mathrm{~cm}$ wide; corelines clasping; fruit flesh cream coloured, soft, crisp, juicy, subacidic; seed cavities 5 , abaxile, obcordate; seeds none to one per cell, none to five per fruit, seed abortion heavy, healthy seeds acute $0.8-0.9 \mathrm{~cm}$ long, $0.4 \mathrm{~cm}$ broad; fruit yield very low; trees annual bearing bloom in $2 \mathrm{nd}$ week of April for 7-10 days; fruit ripens within 100-115 days; harvest time early August.

1.4. Kichhama-trail: Trees medium sized, semierect with extensively branched, round, densely closed crown; flowers pure white, borne in clusters of 5-6; flower spread 4.1-4.5 cm; fruit splashed with red on yellowish background at the time of harvest but blushed or self coloured with bright red on maturity, oblate to nearly spherical, ribbed, irregular, angular, $2.0-2.6 \mathrm{~cm}$ long, $2.9-3.5 \mathrm{~cm}$ across; skin thin smooth covered with thin white powdery bloom which gets rubbed off easily; lenticels on fruit skin few, sparsely placed, depressed or submerged, not very conspicuous; fruit pedicel slendrical, green, pubescent, $1.8-2.5 \mathrm{~cm}$ long, $0.1 \mathrm{~cm}$ thick; stem cavity obtuse, very narrow and shallow, clear, smooth, occasionally lined with thin russet, $0.7-0.9 \mathrm{~cm}$ wide, $0.1-0.3 \mathrm{~cm}$ deep; basin very small, corrugated or mommiform, frequently compressed, $1.5-1.8 \mathrm{~cm}$ wide, $0.1-0.2 \mathrm{~cm}$ deep; calyx persistent, connivent; eye closed; calyx tube cone shaped, $0.3-0.4 \mathrm{~cm}$ deep, $0.2-0.3 \mathrm{~cm}$ wide; persistent stamens placed at the base of calyx tube; pistil point absent; core median or nearly sessile, closed, $1.8-2.0 \mathrm{~cm}$ wide; corelines clasping; fruit flesh yellow, soft, quite juicy and crisp, aromatic, subacidic, seed cavities 5 , axile, obcordate; seeds $1-2$ per cell, $3-5$ per fruit, pointed, $0.7-0.8 \mathrm{~cm}$ long, $0.3-0.4 \mathrm{~cm}$ broad, mostly aborted; fruit-yield quite high; trees strict biennials; bloom in second week of April for 9-12 days; fruit ripens within 170-180 days; harvesting time mid October.

1.5. Sabe-alif: Trees large with widespreading round densely closed head; flowers white, $4.8-6.2 \mathrm{~cm}$ across borne in clusters of 5-7; fruit striped in red against greenish yellow background, oblate, regular, symmetric, occasionally lopsided, uniform, 5.6-6.4 $\times 7.2-7.6 \mathrm{~cm}$ in size; skin thin smooth, waxy, russeted in the cavity; lenticels submerged, areolar, surrounded by a pale halo, conspicuous; fruit pedicel short, thick, dull green with dirty white pubescence $0.6-0.8 \mathrm{~cm}$ long, $0.3 \mathrm{~cm}$ thick; stem cavity acute, $1.5-1.8 \mathrm{~cm}$ deep, $2.4-2.7 \mathrm{~cm}$ wide; basin medium to deep, abrupt, rarely gradual, symmetrical, regular, $2.2-2.8 \mathrm{~cm}$ wide, $0.5-0.7 \mathrm{~cm}$ deep; calyx persistent, reflexed, flat; eye closed; calyx tube cone shaped $0.4-0.5 \mathrm{~cm}$ wide, $0.5-0.7 \mathrm{~cm}$ deep; core median, open, $2.9-3.9 \mathrm{~cm}$ wide; corelines meeting; fruit-flesh white firm with yellow tinge, hard, tough, very juicy, crisp, subacidic; seed cavities 5 , ovate, abaxile; seeds acute to acuminate, 2 per cell, 10 per fruit, dark brown, $0.7-0.8 \mathrm{~cm}$ long, $0.4 \mathrm{~cm}$ wide; blooming period mid April, each tree keeps in bloom for 8-11 days; fruit ripens within 115-125 days; harvest time early to late August.

1.6. Tursh-nawabi: Trees large with round, closed head; branches drooping; flowers white borne in clusters of 5-6, six flowered clusters more frequent, flower 
spread 4.9-5.8 cm; fruit oval to conical, irregular, compressed or sometimes even ribbed, anglular, not uniform, $5.0-7.0 \mathrm{~cm}$ long, $5.0-6.2 \mathrm{~cm}$ across, striped or splashed in pinkish red against greenish yellow background; skin thick, smooth, shining, waxy; lenticels on fruit skin very few, small, inconspicuous, depressed; fruit pedicel brown, pubescent, occasionally clubbed in stem cavity, $0.7-1.5 \mathrm{~cm}$ long, $0.2 \mathrm{~cm}$ thick; stem cavity narrow, acute to acuminate, $1.5-2.0 \mathrm{~cm}$ broad, $1.5-1.9 \mathrm{~cm}$ deep; basin gradual, symmetric, occasionally compressed, wavy to corrugated or even mommiform, $2.0-2.3 \mathrm{~cm}$ wide, $0.3-0.6 \mathrm{~cm}$ deep; calyx persistent, reflexed, divergent, nearly flattened; eye closed or partly open; calyx tube cone shaped, $0.4-0.7 \mathrm{~cm}$ deep, $0.3-0.5 \mathrm{~cm}$ broad; persistent stamens positioned at the middle of calyx tube, protruding through the eye; core median, open, $3.0-3.5 \mathrm{~cm}$ wide; corelines meeting; fruit flesh cream-coloured, soft, quite juicy, crisp, subacidic; seed cavities 5 , abaxile; seeds none to two per cell, $2-7$ per fruit, acute, $0.7-0.9 \mathrm{~cm}$ long, $0.3-0.4 \mathrm{~cm}$ broad, aborted seeds quite frequent; fruit yield fairly good; trees alternate bearers, blooms in mid April for 6-9 days; fruit ripens within 120-140 days; harvesting period mid August to early September.

\section{Pollen mother cell meiosis}

Varieties Lal-farashi, Double-kaseri, Hindwand-rakam, Kichhama-trail, Sabealif and Tursh-nawabi bearing 51 chromosomes, are all triploids, based on x, 17 . Metaphase-I spreads are well scattered and stained and therefore permit detailed analysis. Data collected on chromosome associations during MI, have been presented in Table 1.

The chromosomes pair in twos or threes; $1-20$ chromosomes per cell remain as univalents either because they fail to pair or on account of precocious disjunction. The 220 pollen mother cells scored from the six varieties displayed 50 different patterns of chromosome associations. Frequencies of different associations vary from cell to cell and variety to variety. The most frequently observed patterns are $12 \mathrm{III}+$ $6 \mathrm{II}+3 \mathrm{I}$ in $26.6 \%$ cells of Sabe-alif and $12 \mathrm{III}+5 \mathrm{II}+5 \mathrm{I}$ in $25.0 \%$ cells of Kichhamatrail (Fig. 1).

It is important to note that in all the six varieties a significant number of pollen mother cells (for frequency see Table 7) has an equal number of univalents and bivalents which creates the possibility that these might have been involved in trivalent associations during early prophase (Figs. 1-6). The frequency of such cells is highest in Kichhama-trail $(65.0 \%)$ and least in Sabe-alif $(10.0 \%)$.

The breakup of chromosomes involved in various association is contained in Table 2. The average frequency of different chromosome associations per cell is given in Table 3. The table reveals that univalents, bivalents and trivalents occur in equal proportion in Lal-farashi. The maximum univalent frequency per cell exists in Double-kaseri (Fig. 7); the value is 1.4-1.7 times higher than the number of bi- and trivalents. Trivalent frequency is least in this variety $(7.13 \pm 3.07$ per cell). Kichhama-trail and Tursh-nawabi have the largest number of trivalents ( $1 \frac{1}{2}$ more than the number of bivalents) per cell $(10.17 \pm 2.79$ and $10.0 \pm 2.75)$. A pollen mother cell of Kichhama-trail contained $16 \mathrm{III}+1 \mathrm{II}+1 \mathrm{I}$ (Fig. 3). This configuration is only slightly different from what would be expected in a true autotriploid (17III). 
Table 1. Metaphase I associations in different varieties

\begin{tabular}{|c|c|c|c|c|c|c|c|c|c|}
\hline \multirow[b]{2}{*}{$\begin{array}{c}\text { I } \\
\text { (a) }\end{array}$} & \multirow[b]{2}{*}{$\begin{array}{l}\text { II } \\
\text { (b) }\end{array}$} & \multirow[b]{2}{*}{$\begin{array}{l}\text { III } \\
\text { (c) }\end{array}$} & \multicolumn{6}{|c|}{ Number of pollen mother cells } & \multirow[b]{2}{*}{$\begin{array}{c}\text { Total } \\
\text { no. of } \\
\text { cells } \\
\text { (j) }\end{array}$} \\
\hline & & & $\begin{array}{c}\text { Lal- } \\
\text { farashi } \\
\text { (d) }\end{array}$ & $\begin{array}{c}\text { Double- } \\
\text { kaseri } \\
\text { (e) }\end{array}$ & $\begin{array}{l}\text { Hind- } \\
\text { wand- } \\
\text { rakam } \\
\text { (f) }\end{array}$ & $\begin{array}{l}\text { Kichh- } \\
\text { ama- } \\
\text { trail } \\
\text { (g) }\end{array}$ & $\begin{array}{c}\text { Sabe- } \\
\text { alif } \\
\text { (h) }\end{array}$ & $\begin{array}{c}\text { Tursh- } \\
\text { nawabi } \\
\text { (i) }\end{array}$ & \\
\hline 5 & 5 & $12^{*}$ & - & 2 & 3 & 10 & 1 & 9 & 25 \\
\hline 3 & 6 & 12 & 2 & - & - & 1 & 8 & 3 & 14 \\
\hline 4 & 7 & 11 & 3 & 1 & - & 2 & 4 & 1 & 11 \\
\hline 5 & 8 & 10 & 3 & - & 5 & 2 & - & - & 10 \\
\hline 7 & 7 & $10^{*}$ & - & - & - & 2 & 1 & 6 & 9 \\
\hline 6 & 9 & 9 & 4 & 1 & - & 1 & - & 3 & 9 \\
\hline 3 & 3 & $14^{*}$ & 1 & - & 2 & 3 & - & 3 & 9 \\
\hline 10 & 13 & 5 & 5 & - & 3 & - & - & - & 8 \\
\hline 10 & 7 & 9 & 6 (Fig. 8) & 8) 1 & - & 1 & - & - & 8 \\
\hline 9 & 12 & 6 & 1 & - & 3 & 1 & - & 3 & 8 \\
\hline 7 & 13 & 6 & 1 & - & - & 1 & 2 & 4 & 8 \\
\hline 7 & 10 & 8 & 3 & - & - & 2 & 2 & - & 7 \\
\hline 6 & 6 & $11^{*}$ & 2 & 2 & - & 2 & - & 1 & 7 \\
\hline 9 & 9 & $8^{*}$ & - & - & 1 & 2 & 1 & 2 & 6 \\
\hline 18 & 9 & 5 & 1 & 3 & - & 1 & - & - & 5 \\
\hline 16 & 10 & 5 & - & 2 & 2 & - & - & - & 4 \\
\hline 15 & 9 & 6 & - & 1 & 1 & - & 2 & - & 4 \\
\hline 14 & 8 & 7 & - & - & 4 & - & - & - & 4 \\
\hline 11 & 11 & $6^{*}$ & - & - & 1 & 1 & - & 2 & 4 \\
\hline 10 & 10 & $7^{*}$ & - & - & 1 & 2 & - & 1 & 4 \\
\hline 8 & 11 & 7 & 2 & 1 & - & - & - & 1 & 4 \\
\hline 7 & 4 & 12 & 1 & - & - & 1 & - & 2 & 4 \\
\hline 4 & 4 & $13^{*}$ & 2 & - & - & 1 & - & 1 & 4 \\
\hline 3 & 12 & 8 & - & - & - & - & 2 & 2 & 4 \\
\hline 12 & 15 & 3 & 1 & 1 & - & 1 & - & - & 3 \\
\hline 12 & 9 & 7 & - & - & 3 & - & - & - & 3 \\
\hline 8 & 8 & $9^{*}$ & - & - & - & 2 & - & 1 & 3 \\
\hline 4 & 13 & 7 & - & - & 2 & - & 1 & - & 3 \\
\hline 18 & 12 & 3 & - & 1 & 1 & - & - & - & 2 \\
\hline 13 & 10 & 6 & - & - & 2 & - & - & - & 2 \\
\hline 13 & 7 & 8 & - & 2 & - & - & - & - & 2 \\
\hline 12 & 3 & 11 & 2 & - & - & - & - & - & 2 \\
\hline 4 & 1 & 15 & - & 1 & - & - & 1 & - & 2 \\
\hline 3 & 9 & 10 & - & - & - & - & - & 2 & 2 \\
\hline 2 & 5 & 13 & -. & - & - & - & - & 2 & 2 \\
\hline 20 & 11 & 3 & - & 1 & - & - & - & - & 1 \\
\hline 19 & 10 & 4 & - & 1 & - & - & - & - & 1 \\
\hline 17 & 14 & 2 & - & - & 1 & - & - & - & 1 \\
\hline 17 & 2 & 10 & - & - & - & - & 1 & - & 1 \\
\hline 15 & 6 & 8 & - & - & 1 & - & - & - & 1 \\
\hline 11 & 8 & 8 & - & - & - & - & 1 & - & 1 \\
\hline 9 & 15 & 4 & - & - & - & - & 1 & - & 1 \\
\hline 9 & 6 & 10 & - & 1 & - & - & - & - & 1 \\
\hline 8 & 14 & 5 & - & - & - & - & 1 & - & 1 \\
\hline 6 & 15 & 5 & - & - & - & - & 1 & - & 1 \\
\hline 6 & 12 & 7 & - & - & 1 & - & - & - & 1 \\
\hline 6 & 3 & 13 & - & - & - & - & - & 1 & 1 \\
\hline 5 & 11 & 8 & - & - & 1 & - & - & - & 1 \\
\hline 1 & 1 & $16^{*}$ & - & - & - & 1 & - & - & 1 \\
\hline Tota & of & scored & 40 & 22 & 38 & 40 & 30 & 50 & 220 \\
\hline
\end{tabular}

* PMCs with equal number of uni- and bivalents. Kichhama-trail $(65.0 \%)$; Tursh-nawabi $(42.0 \%)$, Hindwand rakam $(28.5 \%)$, Double-kaseri $(18.2 \%)$, Lal-farashi $(12.5 \%)$, Sabealif $(10.0 \%)$. 
Chromosome segregation is highly erratic (Table 4). Seventy nine segregation patterns have been observed in different PMCs of these varieties. The percentage of cells following $26: 25$ (Fig. 9), distribution is $14.2 \%$ in Kichhama-trail, $14 \%$ in Sabe-alif, $11.0 \%$ in Lal-farashi and $11.1 \%$ in Tursh-nawabi.

Segregation patterns producing nuclei with full haploid or diploid chromosome complement are very rare (Table 4). Cells that follow 26:25 (Fig. 9), 27:24 and

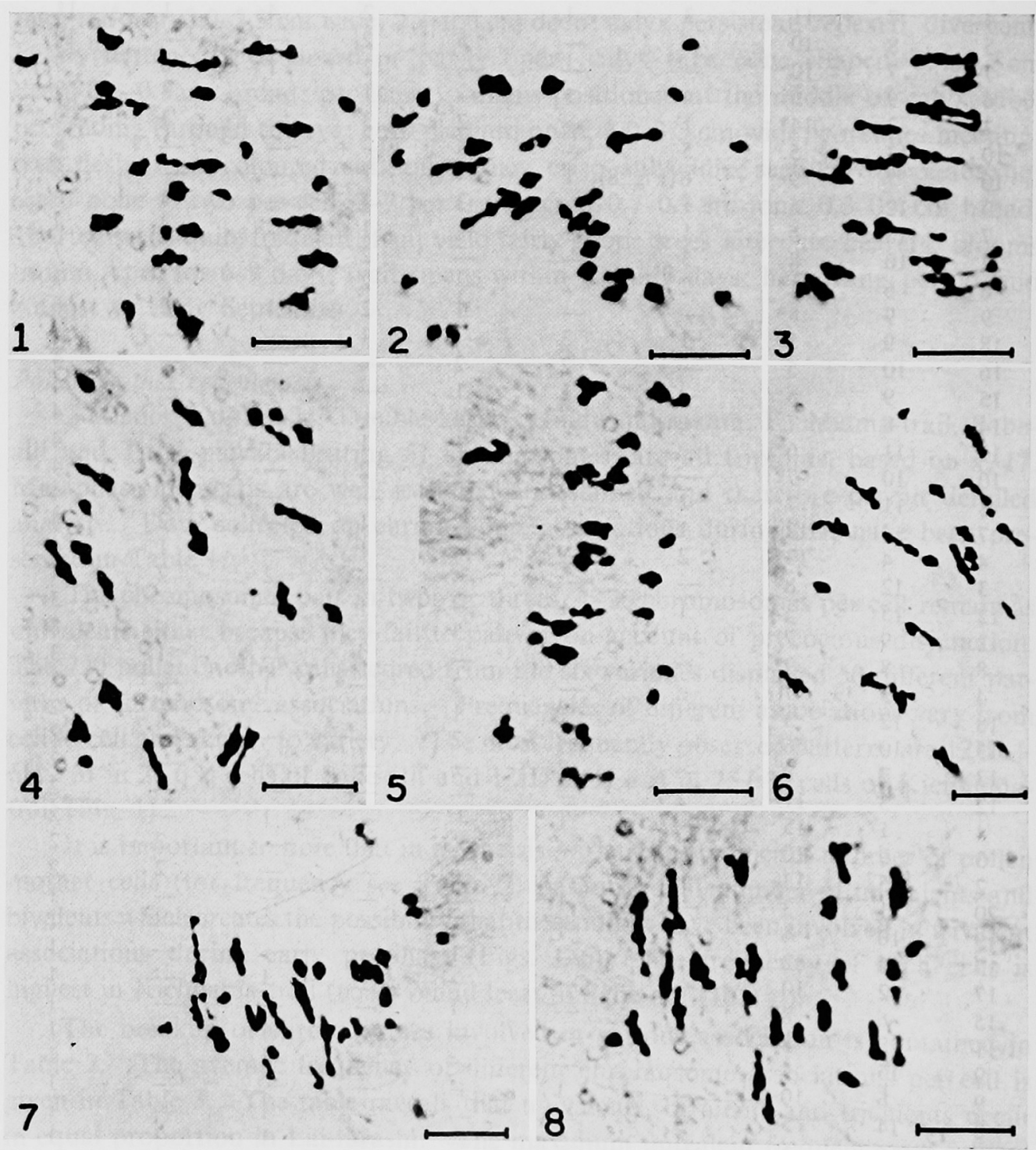

Figs. 1-8. 1, a PMC of Kichhama-trail at metaphase I showing $12 \mathrm{III}+5 \mathrm{II}+5 \mathrm{I} . \mathrm{Bar}=10 \mu \mathrm{m}$. 2, a PMC of Kichhama-trail at mataphase-I showing $6 \mathrm{III}+11 \mathrm{II}+11 \mathrm{I}$. Bar $=10 \mu \mathrm{m}$. 3, a PMC of Kichhama-trail at metaphase I showing $16 \mathrm{III}+1 \mathrm{II}+1 \mathrm{I}$. Bar $=10 \mu \mathrm{m}$. 4, a PMC of Turshnawabi at metaphase I showing $14 \mathrm{III}+3 \mathrm{II}+3 \mathrm{I}$. Bar $=10 \mu \mathrm{m}$. 5, a PMC of sabe-alif at metaphase I showing $12 \mathrm{III}+5 \mathrm{II}+5 \mathrm{I}$. Bar $=10 \mu \mathrm{m}$. 6, a PMC of Hindwand-rakam at metaphase I showing $14 \mathrm{III}+3 \mathrm{II}+3 \mathrm{I}$. Bar $=10 \mu \mathrm{m}$. 7, a PMC of Double-kaseri at metaphase I showing $8 \mathrm{III}+7 \mathrm{II}+13 \mathrm{I}$. Bar $=10 \mu \mathrm{m}$. 8, a PMC of Lal-farashi at metaphase I showing $9 \mathrm{III}+7 \mathrm{II}+$ $10 \mathrm{I}$. $\mathrm{Bar}=10 \mu \mathrm{m}$. 


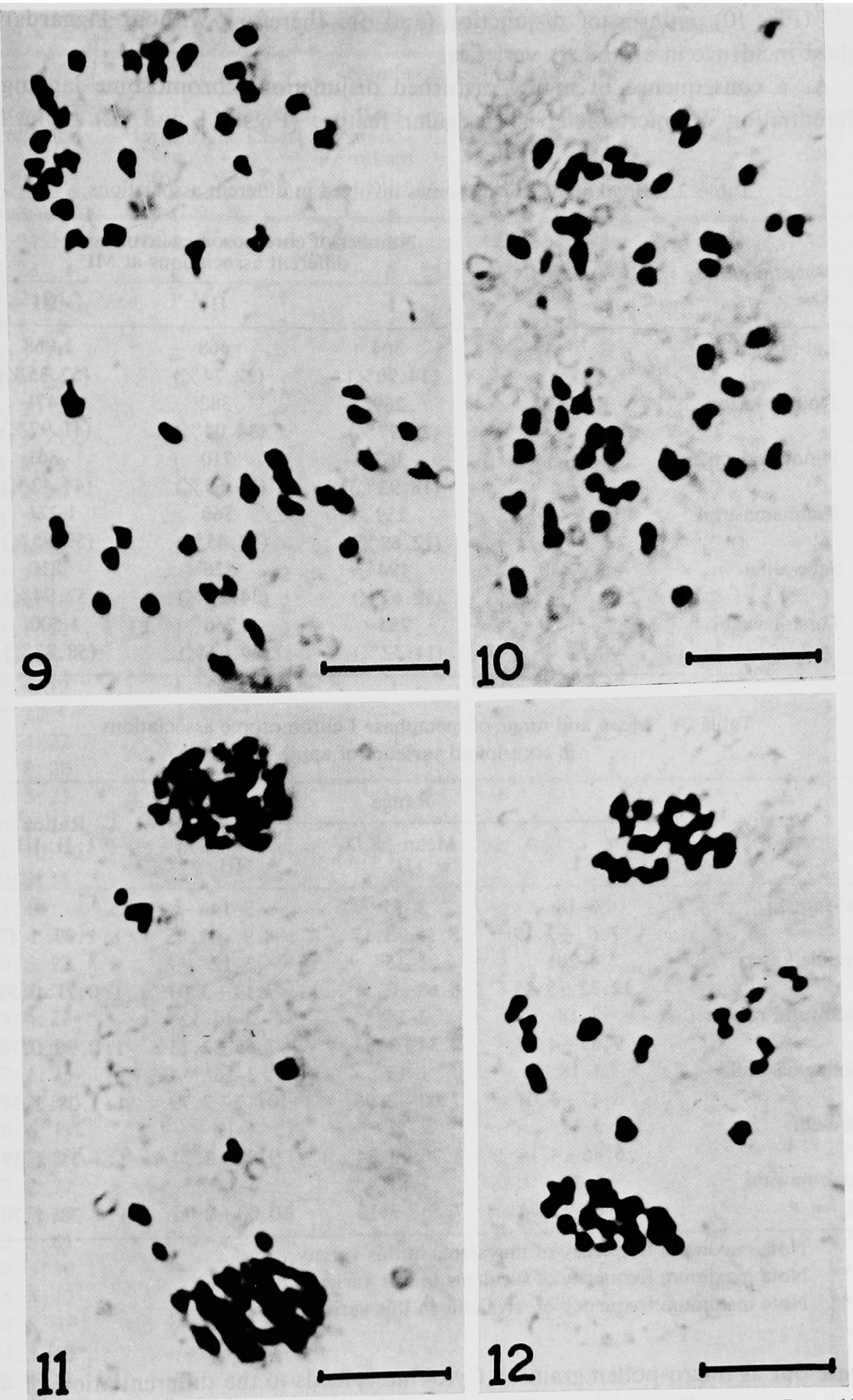

Figs. 9-12. 9, a PMC of Lal-farashi at anaphase I showing 25: 26 segregation. Bar $=10 \mu \mathrm{m}$. 10, a PMC of Kichhama-trail at anaphase I showing 28:23 segregation. Bar $=10 \mu \mathrm{m}$. 11 , a PMC of Lal-farashi showing at least 6 laggards. Bar $=10 \mu \mathrm{m} . \quad 12$, a PMC of Sabe-alif showing 11 laggards. $B a r=10 \mu \mathrm{m}$. 
28:23 (Fig. 10) patterns fof disjunction (and are therefore, without laggards) have highest incidence in all the six varieties.

As a consequence of highly disturbed disjunction, chromosome lagging and differentiation of micronuclei is a regular feature (Figs. 11 and 12). The latter

Table 2. Breakup of chromosomes involved in different associations

\begin{tabular}{|c|c|c|c|c|}
\hline \multirow[t]{2}{*}{ Varietal name } & \multirow{2}{*}{$\begin{array}{l}\text { No. of } \\
\text { PMCs } \\
\text { scored }\end{array}$} & \multicolumn{3}{|c|}{$\begin{array}{l}\text { Number of chromosomes involved in } \\
\text { different associations at MI }\end{array}$} \\
\hline & & I & II & III \\
\hline Lal-farashi & 40 & $\begin{array}{c}304 \\
(14.90 \%)\end{array}$ & $\begin{array}{c}668 \\
(32.74 \%)\end{array}$ & $\begin{array}{c}1,068 \\
(52.35 \%)\end{array}$ \\
\hline Double-kaseri & 22 & $\begin{array}{c}269 \\
(23.97 \%)\end{array}$ & $\begin{array}{c}382 \\
(34.04 \%)\end{array}$ & $\begin{array}{c}471 \\
(41.97 \%)\end{array}$ \\
\hline Hindwand-rakam & 38 & $\begin{array}{c}367 \\
(18.93 \%)\end{array}$ & $\begin{array}{c}710 \\
(36.63 \%)\end{array}$ & $\begin{array}{c}861 \\
(44.42 \%)\end{array}$ \\
\hline Kichhama-trail & 40 & $\begin{array}{c}259 \\
(12.69 \%)\end{array}$ & $\begin{array}{c}560 \\
(27.45 \%)\end{array}$ & $\begin{array}{c}1,221 \\
(59.85 \%)\end{array}$ \\
\hline Sabe-alif & 30 & $\begin{array}{c}194 \\
(12.67 \%)\end{array}$ & $\begin{array}{c}526 \\
(34.27 \%)\end{array}$ & $\begin{array}{c}810 \\
(52.94 \%)\end{array}$ \\
\hline Tursh-nawabi & 50 & $\begin{array}{c}294 \\
(11.52 \%)\end{array}$ & $\begin{array}{c}756 \\
(29.64 \%)\end{array}$ & $\begin{array}{c}1,500 \\
(58.82 \%)\end{array}$ \\
\hline
\end{tabular}

Table 3. Mean and range of metaphase I chromosome associations in six triploid varieties of apple

\begin{tabular}{|c|c|c|c|c|}
\hline \multirow{2}{*}{ Variety } & \multicolumn{3}{|c|}{ Range } & \multirow{2}{*}{$\begin{array}{l}\text { Ratios } \\
\text { I: II: III }\end{array}$} \\
\hline & I & $\underset{\text { II }}{\operatorname{Mean}} \pm$ S.D & III & \\
\hline Lal-farashi & $3-18$ & $3-15$ & $3-14$ & \\
\hline & $7.6 \pm 3.19$ & $8.35 \pm 3.17$ & $8.9 \pm 2.65$ & $1: 1.09: 1.17$ \\
\hline Double-kaseri & $4-20 *$ & $5-15$ & $3-12$ & \\
\hline Hindwand-rakam & $\begin{array}{c}12.22 \pm 5.35 \\
3-18\end{array}$ & $\begin{array}{c}8.68 \pm 2.51 \\
3-14^{* *}\end{array}$ & $\begin{array}{l}7.13 \pm 3.07 \\
2-14\end{array}$ & $1: 0.71: 0.58$ \\
\hline Kichhama-trail & $\begin{array}{c}9.65 \pm 4.77 \\
1-18\end{array}$ & $\begin{array}{c}9.34 \pm 2.83 \\
1-15\end{array}$ & $\begin{array}{c}7.55 \pm 2.73 \\
3-16^{* * *}\end{array}$ & $1: 0.96: 0.78$ \\
\hline Sabe-alif & $\begin{array}{c}6.47 \pm 3.07 \\
3-17\end{array}$ & $\begin{array}{c}7.00 \pm 2.96 \\
2-15\end{array}$ & $\begin{array}{c}10.17 \pm 2.79 \\
4-12\end{array}$ & $1: 1.08: 1.57$ \\
\hline Tursh-nawabi & $\begin{array}{c}6.46 \pm 4.16 \\
2-11 \\
5.88 \pm 2.34\end{array}$ & $\begin{array}{c}8.76 \pm 3.34 \\
3-13 \\
7.56 \pm 3.12\end{array}$ & $\begin{array}{c}9.00 \pm 2.74 \\
6-14^{* * *} \\
10.00+2.75\end{array}$ & $1: 1.35: 1.39$ \\
\hline
\end{tabular}

* Note maximum frequency of univalents in this variety.

** Note maximum frequency of bivalents in this variety.

*** Note maximum frequency of trivalents in this variety.

separate out as micro-pollen grains. Cytokinesis leads to the differentiation of 4-8 microspores per pollen mother cell.

Data on pollen stainability and dimensions are given in Table 5. 
Table 4. Anaphase disjunction patterns in six triploid varieties

\begin{tabular}{|c|c|c|c|c|c|c|c|}
\hline \multirow[b]{2}{*}{$\begin{array}{l}\text { Patterns of } \\
\text { segregation } \\
\text { (a) }\end{array}$} & \multicolumn{7}{|c|}{ Number of pollen mother cells } \\
\hline & $\begin{array}{c}\text { Lal- } \\
\text { farashi } \\
\text { (b) }\end{array}$ & $\begin{array}{c}\text { Double- } \\
\text { kaseri } \\
\text { (c) }\end{array}$ & $\begin{array}{l}\text { Hind- } \\
\text { wand- } \\
\text { rakam } \\
\text { (d) }\end{array}$ & $\begin{array}{l}\text { Kichh- } \\
\text { ama- } \\
\text { trail } \\
\text { (e) }\end{array}$ & $\begin{array}{l}\text { Sabe- } \\
\text { alif } \\
\text { (f) }\end{array}$ & $\begin{array}{l}\text { Tursh- } \\
\text { nawabi } \\
\text { (g) }\end{array}$ & $\begin{array}{l}\text { Total } \\
\text { no. of } \\
\text { cells } \\
\text { (h) }\end{array}$ \\
\hline $26: 25$ & 4 & - & 1 & 4 & 4 & 4 & 17 \\
\hline $27: 24$ & 4 & - & 2 & 4 & 2 & 4 & 16 \\
\hline $28: 23$ & 1 & - & 6 & 4 & 3 & 1 & 15 \\
\hline $25: 2: 24$ & 1 & 1 & 1 & 2 & 2 & - & 7 \\
\hline $24: 6: 21$ & - & 2 & 1 & 2 & - & 1 & 6 \\
\hline $25: 5: 21$ & 2 & - & 1 & 2 & - & - & 5 \\
\hline $25: 1: 25$ & 2 & 1 & 1 & - & - & 1 & 5 \\
\hline $31: 20$ & - & - & - & 1 & 2 & 2 & 5 \\
\hline $27: 3: 21$ & - & - & 1 & 1 & - & 3 & 5 \\
\hline $24: 8: 19$ & 1 & 2 & 1 & - & - & 1 & 5 \\
\hline $29: 2: 20$ & - & 1 & - & - & 1 & 2 & 4 \\
\hline $24: 5: 22$ & - & - & - & - & 1 & 3 & 4 \\
\hline $23: 6: 22$ & 1 & - & - & - & 1 & 2 & 4 \\
\hline $30: 7: 14$ & - & 2 & - & - & 1 & - & 3 \\
\hline $29: 4: 18$ & - & 2 & - & 1 & - & - & 3 \\
\hline $33: 1: 17^{*}$ & 1 & 2 & - & - & - & - & 3 \\
\hline $30: 21$ & - & - & - & - & 2 & 1 & 3 \\
\hline $28: 4: 19$ & 1 & - & 1 & - & - & 1 & 3 \\
\hline $29: 22$ & 3 & - & - & - & - & - & 3 \\
\hline $25: 4: 22$ & - & 1 & 1 & - & - & 1 & 3 \\
\hline $23: 8: 20$ & 1 & 1 & - & - & 1 & - & 3 \\
\hline $23: 5: 23$ & - & 1 & 1 & - & 1 & - & 3 \\
\hline $26: 1: 24$ & 1 & 1 & 1 & - & - & - & 3 \\
\hline $21: 10: 20$ & 1 & - & - & - & 1 & 1 & 3 \\
\hline $30: 3: 18$ & - & - & 1 & - & 1 & - & 2 \\
\hline $33: 18$ & 1 & - & 1 & - & - & - & 2 \\
\hline $27: 4: 20$ & 1 & - & - & - & - & 1 & 2 \\
\hline $26: 3: 22$ & - & - & - & 1 & 1 & - & 2 \\
\hline $26: 2: 23$ & - & - & 1 & 1 & - & - & 2 \\
\hline $24: 4: 23$ & - & 1 & - & 1 & - & - & 2 \\
\hline $25: 7: 19$ & - & - & - & - & 1 & 1 & 2 \\
\hline $27: 1: 23$ & - & - & - & - & 1 & 1 & 2 \\
\hline $28: 5: 18$ & - & - & 2 & - & - & - & 2 \\
\hline $26: 6: 19$ & - & 1 & 1 & - & - & - & 2 \\
\hline $24: 7: 20$ & 1 & 1 & - & - & - & - & 2 \\
\hline $27: 2: 22$ & - & - & 1 & 1 & - & - & 2 \\
\hline $23: 9: 19$ & 1 & 1 & - & - & - & - & 2 \\
\hline $22: 8: 21$ & - & - & 2 & - & - & - & 2 \\
\hline $36: 5: 10$ & - & 1 & - & - & - & - & 1 \\
\hline $35: 4: 12$ & - & 1 & - & - & - & - & 1 \\
\hline $34: 8: 9^{*}$ & - & 1 & - & - & - & - & 1 \\
\hline $34: 7: 10^{*}$ & - & - & 1 & - & - & - & 1 \\
\hline $32: 1: 18$ & - & 1 & - & - & - & - & 1 \\
\hline $32: 2: 17^{*}$ & - & - & - & - & 1 & - & 1 \\
\hline $32: 19$ & - & - & - & - & 1 & - & 1 \\
\hline $31: 4: 16$ & - & - & - & - & - & 1 & 1 \\
\hline $31: 9: 11$ & 1 & - & - & - & - & - & 1 \\
\hline
\end{tabular}


Table 4. (continued)

\begin{tabular}{|c|c|c|c|c|c|c|c|}
\hline \multirow[b]{2}{*}{$\begin{array}{l}\text { Patterns of } \\
\text { segregation } \\
\text { (a) }\end{array}$} & \multicolumn{7}{|c|}{ Number of pollen mother cells } \\
\hline & $\begin{array}{l}\text { Lal- } \\
\text { farashi } \\
\text { (b) }\end{array}$ & $\begin{array}{c}\text { Double- } \\
\text { kaseri } \\
\text { (c) }\end{array}$ & $\begin{array}{l}\text { Hind- } \\
\text { wand- } \\
\text { rakam } \\
\text { (d) }\end{array}$ & $\begin{array}{l}\text { Kichh- } \\
\text { ama- } \\
\text { trail } \\
\text { (e) }\end{array}$ & $\begin{array}{l}\text { Sabe- } \\
\text { alif } \\
\text { (f) }\end{array}$ & $\begin{array}{c}\text { Tursh- } \\
\text { nawabi } \\
\text { (g) }\end{array}$ & $\begin{array}{l}\text { Total } \\
\text { no. of } \\
\text { cells } \\
\text { (h) }\end{array}$ \\
\hline $31: 2: 18$ & - & - & - & - & 1 & - & 1 \\
\hline $31: 3: 17^{*}$ & - & 1 & - & - & - & - & 1 \\
\hline $30: 5: 16$ & - & - & 1 & - & - & - & 1 \\
\hline $30: 2: 19$ & 1 & - & - & - & - & - & 1 \\
\hline $29: 5: 17^{*}$ & - & - & - & - & 1 & - & 1 \\
\hline $29: 1: 21$ & - & - & - & - & 1 & - & 1 \\
\hline $29: 7: 15$ & - & 1 & - & - & - & - & 1 \\
\hline $28: 9: 14$ & - & 1 & - & - & - & - & 1 \\
\hline $28: 12: 11$ & - & 1 & - & - & - & - & 1 \\
\hline $28: 6: 17^{*}$ & - & - & 1 & - & - & - & 1 \\
\hline $28: 3: 20$ & - & - & - & - & - & 1 & 1 \\
\hline $28: 7: 16$ & - & - & - & - & - & 1 & 1 \\
\hline $28: 2: 21$ & - & - & - & 1 & - & - & 1 \\
\hline $28: 1: 22$ & 1 & - & - & - & - & - & 1 \\
\hline $26: 7: 18$ & - & - & - & - & 1 & - & 1 \\
\hline $26: 5: 20$ & - & - & - & 1 & - & - & 1 \\
\hline $26: 4: 21$ & - & - & - & 1 & 一 & - & 1 \\
\hline $25: 3: 23$ & - & - & 1 & - & - & - & 1 \\
\hline $24: 12: 15$ & - & 1 & - & - & - & - & 1 \\
\hline $24: 13: 14$ & - & 1 & - & - & - & - & 1 \\
\hline $24: 3: 24$ & - & - & - & - & 1 & - & 1 \\
\hline $24: 6: 21$ & 1 & - & - & - & - & - & 1 \\
\hline $23: 7: 21$ & 1 & - & - & - & - & - & 1 \\
\hline $22: 7: 22$ & 1 & - & - & - & - & - & 1 \\
\hline $22: 11: 18$ & - & - & - & - & 1 & - & 1 \\
\hline $22: 9: 20$ & 一 & - & - & - & - & 1 & 1 \\
\hline $21: 13: 17^{*}$ & 1 & - & -- & - & - & - & 1 \\
\hline $21: 15: 15$ & - & 1 & - & - & - & 一 & 1 \\
\hline $20: 12: 19$ & 1 & - & - & - & - & - & 1 \\
\hline $20: 11: 20$ & - & - & - & - & - & 1 & 1 \\
\hline $\begin{array}{l}\text { Total no. } \\
\text { of pMCs }\end{array}$ & 36 & 32 & 32 & 28 & 34 & 36 & 198 \\
\hline
\end{tabular}

* Nuclei with full haploid or diploid complement of 17 or 34 chromosomes.

Table 5. Pollen stainability and dimensions

\begin{tabular}{lcc}
\hline \multicolumn{1}{c}{ Variety } & $\begin{array}{c}\text { Stainable } \\
\text { pollen } \\
(\%)\end{array}$ & $\begin{array}{c}\text { Average length of } \\
\text { the equatorial axis } \\
\text { of stainable pollen } \\
\text { (in } \mu \mathrm{m})\end{array}$ \\
\hline Tursh-nawabi & 68.24 & 42.17 \\
Dundwand-rakam & 60.42 & 41.86 \\
Sabe-alif & 51.05 & 39.13 \\
Double-kaseri & 41.25 & 42.25 \\
Lal-farashi & 40.08 & 42.05 \\
Kichhama-trail $^{*}$ & 14.20 & 40.63 \\
\hline
\end{tabular}

* More than $85 \%$ pollen of this variety is inviable. 


\section{Discussion}

In apple it is customary to assess the impact of polyploidy on fruit characteristics through intervarietal comparisons (see Singh and Wafai, in press). In our previous publication (Koul et al. in press) which deals with triploid varieties, Rikhalsaharanpuri, Kharoo and Acc. 1877, it was pointed out that triploids do not have any edge over the diploids but they are certainly superior to the tetraploids. In order to see whether this holds true even for the six triploids studied now, comparisons have been drawn between these and some diploid and tetraploid cultivars.

Table 6 reveals that some diploid varieties bear larger and more tasteful fruits than these triploids. The triploid fruit is generally subacidic. Nevertheless, they outshine the tetraploid Khatoon-trail. Kichhama-trail repersents an exception. Despite its triploid nature, the fruit is less than half the fruit size of $4 \mathrm{x}$ Khatoontrail. Smaller size notwithstanding, Kichhama-trail maintains its superiority over Khatoon-trail in taste.

Table 6. Comparison of the tree, floral and fruit characteristics of diploid and polyploid cultivars of apple grown in Kashmir Valley

\begin{tabular}{|c|c|c|c|}
\hline Character & Diploid $^{+}$ & Triploid $^{++}$ & Tetraploid $^{+++}$ \\
\hline Tree size & medium & $\begin{array}{l}\text { small to } \\
\text { large }\end{array}$ & medium \\
\hline Blooming period: (days) & $5-13$ & $6-12$ & $4-8$ \\
\hline Spread of flower: $(\mathrm{cm})$ & $3.0-6.5$ & $3.2-6.0$ & $3.0-4.5$ \\
\hline Diameter of the fruit $(\mathrm{cm})$ & $2.0-11.0$ & $3.0-8.5$ & $5.0-5.9$ \\
\hline Height of the fruit $(\mathrm{cm})$ & $2.0-10.0$ & $2.0-7.5$ & $4.2-5.0$ \\
\hline Taste of the fruit & $\begin{array}{l}\text { subacidic } \\
\text { or sweet }\end{array}$ & subacidic & subacidic \\
\hline $\begin{array}{l}\text { No. of days required for harvest } \\
\text { Bearing quality }\end{array}$ & $\begin{array}{l}65-190 \\
\text { annual or } \\
\text { biennial }\end{array}$ & $\begin{array}{l}100-180 \\
\text { binenial }\end{array}$ & $\begin{array}{l}85-95 \\
\text { biennial }\end{array}$ \\
\hline
\end{tabular}

+ Data represent an average of 60 diploid varieties.

+ Triploid varieties under reference.

++ Data pertaining to Khatoon-trail.

Singh and Wafai (in press) have convincingly proved that impact of polyploidy on fruit characteristics can be estimated better through intravarietal rather than intervarietal comparisons because in the latter procedure it is difficult to resolve whether size difference is an index of polyploidy or varietal difference. Our observations (Singh and Wafai in press) on variety Hazratbali indicate that triploidy improves fruit quality even at intravarietal level. Triploid Hazratbali II excels its diploid counterpart Hazratbali I, in almost all qualities of the fruit including colour and taste.

Nature of triploidy: The usefulness of pairing behaviour in unravelling the origin and ancestry of polyploids is too well known (Moffett 1931, Roscoe 1934). PMC meiosis of triploid apple is characterised by high frequency of trivalents. Moreover, every variety has a significant number of PMC's carrying an equal number of uni- and bivalents (Table 7) which raises the possiblility that they might have been 
involved in trivalent associations during prophase. In that case the actual frequency of trivalents will exceed the observed frequency. Formation of so many trivalents speaks of sufficient homology between the constituent genomes and suggests autoor at best segmental allotriploid nature of these varieties.

Comparison of the data on meiotic pairing of the four triploid varieties studied earlier (Koul et al. in press and Singh and Wafai in press), as also the six varieties constituting the present test material reveals that they vary a good deal in the degree of homology among their constituent genomes (Table 7). Hazratbali II, Rikhalsaharanpuri and Kichhama-trail bear as many as 17 or 16 trivalents per cell (Fig. 3) which is the maximum theoretically expected in autotriploids. Average trivalent frequency per cell is $10.77 \pm 3.66$ (Rikhal-saharanpuri), $10.17 \pm 2.79$ (Kichhamatrail), $9.88 \pm 3.64$ (Hazratbali II) and $10.00 \pm 2.75$ (Tursh-nawabi). Such pairing behaviour testifies the strong homology among the three genomes of these varieties. Acc. 1877 also falls in this very category; although the maximum number of trivalents formed per cell does not exceed thirteen (Koul et al. in press), the average trivalent

Table 7. Comparisons of pairing of various varieties

\begin{tabular}{lccl}
\hline \multicolumn{1}{c}{ Variety } & $\begin{array}{c}\text { Maximum number } \\
\text { of trivalents } \\
\text { per cell }\end{array}$ & $\begin{array}{c}\% \text { cells bearing } \\
\text { uni- and bivalents } \\
\text { in equal number }\end{array}$ & Authority \\
\hline Hazratbali II & 17 & 32.0 & Singh and Wafai in press \\
Rikhal-saharanpuri & 17 & 18.6 & Koul et al. in press \\
Kichhama trail & 16 & 65.0 & Present work \\
Tursh-nawabi & 14 & 42.0 & Present work \\
Hindwand-rakam & 14 & 28.5 & Present work \\
Lal-farashi & 14 & 12.5 & Present work \\
Acc. 1877 & 13 & 62.5 & Koul et al. in press \\
Sabe-alif & 12 & 10.0 & Present work \\
Double kaseri & 12 & 18.2 & Present work \\
Kharoo & 8 & 8.0 & Koul et al. in press \\
\hline
\end{tabular}

frequency $(10.31 \pm 1.52)$ overlaps that of Rikhal-saharanpuri and Kichhama-trail. That, the maximum number of trivalent associations may be even more than 13 in this variety is indicated by the fact that over $62 \%$ PMCs bear uni- and bivalents in equal numbers which might have been involved as trivalents during prophase. Double-kaseri and Kharoo differ from other triploids in this respect. In Doublekaseri univalents outnumber other chromosome associations $(12.22 \pm 5.35$ per cell), and in Kharoo bivalents represent the dominant association (13.21 \pm 2.4 per cell). Nevertheless both forms have some trivalents as well; $7.13 \pm 3.07$ and $5.88 \pm 1.45$ respectively. These facts speak for weak intergenomal homology.

The remaining varieties, namely Lal-farashi, Hindwand-rakam and Sabealif seem to lie in between these two categories in respect of the degree of differentiation between their genomes.

\section{Summary}

Six triploid varieties of cultivated apple (Malus pumila Mill.) namely Lal- 
farashi, Double-kaseri, Hindwand-rakam, Kichhama trail, Sabe-alif and Turshnawabi having $2 n=51$ have been recovered from different orchards of Kashmir Valley. Although not as good as the diploids, they are far superior to the tetraploid variety Khatoon-trail in their fruit quality. Data on the chromosome pairing reveals intergenomal homology suggesting their auto- or segmental allotriploid nature. The six triploids exhibit varying degrees of intergenomal differentiation.

\section{Acknowledgements}

Thanks are due to the Head, Department of Biosciences for providing the necessary laboratory facilities. The authors wish to put on record the help rendered by Dr. Karan Singh, M. P., Jyoti Pvt. Ltd. orchards, Boulevard, Srinagar, M/s Malik Orchards, Khounmoh (Kashmir) and Mr. A. N. Bhat, Nehru Memorial Botanical Gardens, Chasma Shahi, Srinagar by allowing us free access to their orchards. Without thier help the work could not have been possible. Financial assistance received from the University Grants Commission, New Delhi, is gratefully acknowledged.

\section{Literature cited}

Einset, J. and Imhofe, B. 1947. Chromosome numbers of apple varieties and sports. I. Proc. Amer. Soc. Hort. Sci. 50: 45-50.

- and - 1949. Chromosome numbers of apple varieties and sports II. Proc. Amer. Soc. Hort. Sci. 53: 197-201.

- and Lamb, B. 1951. Chromosome numbers of apple varieties and sports III. Proc. Amer. Soc. Hort. Sci. 58: 103-108.

Hathwala, M. S. 1966. Cytomorphological studies of Indian cultivated varieties of apple grown in Kulu Valley. A Ph. D. Thesis submitted to Punjab Univ. Chandigarh, India.

Hunter, A. W. S. and Walker, G. W. R. 1955. Chromosome numbers in apples. Canada Cent. Expt. Farm. Hort. Div. Prog. Rept. 1949-53: 67-69.

Koul, A. K., Singh, R. and Wafai, B. A. Assessment of the germ-plasm of apple (Malus pumila Mill.) grown in Kashmir II. Cytology of Rikhal saharanpuri, Acc. 1877 and Kharoo. Cytologia (in press).

Lespinasse, Y., Alston, F. H. and Watkins, R. 1976. Cytological techniques for use in apple breeding. Ann. Appl. Biol. 82: 349-353.

Moffett, A. A. 1931. A preliminary account of chromosome behaviour in the Pomoideae. J. Pom. and Hort. Sci. 9: 100-110.

Pratt, C. Joh, S. P., Way, R. D. and Einset, J. 1978. Chromosome numbers of apple species, cultivars and sports V. J. Amer. Soc. Hort. Sci. 103: 690-693.

Roscoe, M. V. 1934. The chromosomal constitution of certain cultivated apple varieties. J. Genetics 28: 157-167.

Shanti, B., Pratt, C. and Einset, J. 1963. Chromosome number of apple varieties and sports IV. Proc. Amer. Soc. Hort. Sci., 82: 56-63.

Singh, R. and Wafai, B. A. Intravarietal polyploidy in the apple (Malus pumila Mill). Cultivar Hazratbali. Euphytica (in press). 